\title{
Multi-objective Test Generation for Software Product Lines
}

\author{
Christopher Henard \\ SnT, University of Luxembourg \\ Luxembourg, Luxembourg \\ Luxembourg, Luxembourg \\ Mike Papadakis \\ SnT, University of Luxembourg \\ Gilles Perrouin \\ Jacques Klein \\ SnT, University of Luxembourg \\ Luxembourg, Luxembourg \\ jacques.klein@uni.lu \\ Yves Le Traon \\ SnT, University of Luxembourg \\ Luxembourg, Luxembourg \\ yves.letraon@uni.lu
}

\begin{abstract}
Software Products Lines (SPLs) are families of products sharing common assets representing code or functionalities of a software product. These assets are represented as features, usually organized into Feature Models (FMs) from which the user can configure software products. Generally, few features are sufficient to allow configuring millions of software products. As a result, selecting the products matching given testing objectives is a difficult problem.

The testing process usually involves multiple and potentially conflicting testing objectives to fulfill, e.g. maximizing the number of optional features to test while at the same time both minimizing the number of products and minimizing the cost of testing them. However, most approaches for generating products usually target a single objective, like testing the maximum amount of feature interactions. While focusing on one objective may be sufficient in certain cases, this practice does not reflect real-life testing situations.

The present paper proposes a genetic algorithm to handle multiple conflicting objectives in test generation for SPLs. Experiments conducted on FMs of different sizes demonstrate the effectiveness, feasibility and practicality of the introduced approach.
\end{abstract}

\section{Categories and Subject Descriptors}

D.2.5 [Software Engineering]: Testing and Debugging

\section{General Terms}

Algorithms, Experimentation

\section{Keywords}

Software Product Lines, Test Generation, Multi-objective Optimization, Genetic Algorithms, Feature Models

\footnotetext{
*FNRS Postdoctoral Researcher.
}

Permission to make digital or hard copies of all or part of this work for personal or classroom use is granted without fee provided that copies are not made or distributed for profit or commercial advantage and that copies bear this notice and the full citation on the first page. To copy otherwise, to republish, to post on servers or to redistribute to lists, requires prior specific permission and/or a fee.

SPLC 2013, August 26 - 30 2013, Tokyo, Japan

Copyright 2013 ACM 978-1-4503-1968-3/13/08 ...\$15.00.

\section{INTRODUCTION}

The software industry is increasingly building software families consisting of similar systems with many variations 5], called variants. Such families are known as Software Product Lines (SPLs). A Software Product Line (SPL) has been defined as a set of software-intensive systems that share a common, managed set of features satisfying the specific needs of a particular market segment or mission and that are developed from a common set of core assets in a prescribed way 3]. Software Product Line Engineering (SPLE) 27 is a software development paradigm designed to handle these variants. This approach results in many benefits, like reducing the maintenance effort, the software development costs and the time of products to market 7]. The features of a SPL are usually organized into a Feature Model (FM) 17 from which the user can configure and derive 28 tailored software products.

Testing a SPL is a inherent difficult task 21]. Indeed, only few features are sufficient to enable the configuration of thousands or millions of variants. For instance, a FM of a video player with 71 features 22 allows configuring more than $1.65 \times 10^{13}$ different products. Therefore, testing all the products of a SPL with an acceptable cost is almost unmanageable. To overcome this problem, approaches have been proposed to reduce the number of products to test 8 . For instance, Combinatorial Interaction Testing (CIT) 4 has been identified as a relevant approach to sample the configuration space. This technique is based on the observation that most of the faults are due to the interaction between a small number of features. As a result, only the products exercising all the interactions between any $t$ features ( $t$-wise) should be tested. This approach has been adapted to SPL testing, e.g. $15,25$.

In real-life situations, selecting the products to test is a multi-dimensional problem. Indeed, the testing process usually involves multiple and possibly conflicting objectives, like generating the products to test with respect to $t$-wise but at the same time minimizing the number of products to test, maximizing the number of optional features exercised and minimizing the cost of testing these products. Such requirements necessitate a trade-off between several testing objectives. However, most of the approaches selecting relevant products to be tested target a single objective at a time. While this may be sufficient in certain cases, this method does not reflect real-life testing situations and constraints. In view of this, this paper proposes a genetic algorithm to 
handle multiple test objectives for SPLs. The approach is validated through a case study conducted on $8 \mathrm{FMs}$.

The remainder of this paper is organized as follows. Section 2 presents the concepts and the motivation underlying the proposed approach. Section 3 details the introduced algorithm to solve multi-objective test generation for SPLs. Section 4 reports on experiments. Finally, Section 5 discusses related work and Section 6 concludes the paper.

\section{BACKGROUND AND MOTIVATION}

This section introduces some notations and concepts used in this paper and motivates the proposed approach.

\subsection{Feature Models and Products}

Feature Models (FMs) represent the features and the constraints linking the features of a SPL. They are usually represented through a Feature Diagram (FD) [6]. Figure 1 depicts an example of FD of a mobile phone product line of 10 features and the dependencies between the features 2. For instance, the presence of the Camera feature requires the High resolution one to be present too.

A FM can easily be translated to logic 23. Doing so results in a boolean formula where its variables represent the features of the FM. A satisfiability (SAT) solver can then be used to generate valid configurations by using this formula. A configuration of a FM is a set of features that are proposed by the software product. The variables of the formula are assigned true for the selected features of the product and false for the others. The configuration is said to be valid if the formula is satisfied, i.e. its evaluation is true. For instance, the following configuration satisfies the formula of the example FM:

$$
C=\{\text { Mobile Phone, Calls, Screen, Basic }\} .
$$

In this configuration, the Mobile phone, Calls and Basic screen variables are assigned to true, while the other variables are assigned to false. Therefore, the configuration is valid since all the constraints of the $\mathrm{FM}$ are fulfilled.

We define as a product the set of selected and unselected features that characterize this product [12]. Thus, if we consider a FM of $n$ features, one product $P$ is represented as $\left\{ \pm f_{1}, \ldots, \pm f_{n}\right\}$, where $+f_{i}$ indicates a feature which is selected by this product, and $-f_{i}$ an unselected one. Like for configurations, a product is said to be valid if it satisfies the constraints of the FM, i.e. its formula. For instance, the following product $P$ associated to the configuration $C$ is valid:

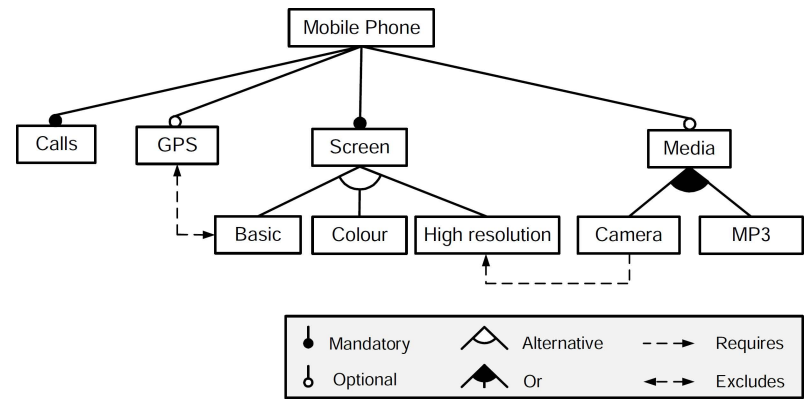

Figure 1: A Feature Diagram of a Mobile Phone Product Line 2].

$$
P=\{+ \text { Mobile Phone },+ \text { Calls },-G P S,+ \text { Screen },+ \text { Basic },
$$$$
\text { -Colour,-High Resolution,-Media,-Camera,-MP3\}. }
$$

\subsection{Test Suites, Cost and Pairwise Coverage}

In this paper, a product corresponds to a test case of a SPL and a test suite to a set of $m$ products. To simplify, we will refer to products as tests.

In an attempt to take into account the testing cost of products, some assumptions are made. We assume that the testing effort of each product is related to the number of features that it contains. Additionally, each feature requires a different amount of resources in order to be tested. To this end, a value representing an estimate of its testing cost is assigned to each feature. Thus, the cost of testing one product is assumed to be equal to the sum of the cost of the features that it is composed of. More formally, if $c_{i}$ denotes the cost of the feature $f_{i}$, a product $P_{j}=\left\{ \pm f_{1}, \ldots, \pm f_{n}\right\}$ has a cost $C_{t}$ equals to:

$$
C_{t}\left(P_{j}\right)=\sum_{i=1}^{n} p(i) c(i), \text { where } p(i)= \begin{cases}1 & \text { if }+f_{i} \\ 0 & \text { if }-f_{i}\end{cases}
$$

The cost of a test suite is the sum of the cost of the $m$ products that it is composed of. Thus, the cost of $x=$ $\left\{P_{1}, \ldots, P_{m}\right\}$ is given by:

$$
\operatorname{cost}(x)=\sum_{j=1}^{m} C_{t}\left(P_{j}\right) .
$$

Putting a cost to each feature transforms the initial FM into an attributed FM 24. A similar way of representing the cost of features and products can be found in 24.

Finally, pairwise is a testing technique that focuses on the interactions between any two features of a SPL 26]. Such an interaction is called a pair of features. Pairwise coverage denotes the ability of a test suite to cover all the pairs of features that exist in a SPL. In this paper, pairwise coverage is measured as the number of pairs covered by the products of the test suite.

\subsection{Motivation}

In practice, software development introduces several constraints on the actual testing process 7. Constraints like occupying a specific amount of resources, meeting a specific budget or finishing the process on time are such examples. To meet these needs, it may be necessary to suspend the testing process before testing all the selected products. In such a case, it is required to minimize the process cost by maximizing the level of testing thoroughness at the same time. This is a difficult task due to the following two problems to face with: $a$ ), in the SPL context, not all the products (as defined in Section 2.1) are necessarily valid. This fact, makes the test selection a hard problem [25]. The problem is so hard that most of the existing approaches are encountering difficulties in selecting products to test 25. $b)$, among the valid products, the aforementioned objectives are competitive, i.e. minimizing the cost reduces the quality of testing and vice versa. This is analogous to test suite minimization, which is known to be a hard problem [29] and it is clearly escalated in the presence of the a) problem.

Addressing both these problems at the same time makes the test selection task challenging. In addition, the practical need of dealing with these problems simultaneously motivates the suggestion of such an approach. In view of this, the 
present paper introduces a novel approach avoiding invalid products and capable of dealing with multiple objectives at the same time. Indeed, it makes a combined use of constraint solving and multi-objective optimization techniques to fulfill the test selection goals. The proposed approach is validated on a set of real feature models dealing with the following objectives:

1. Maximizing the pairwise coverage,

2. Minimizing the number of products selected,

3. Minimizing the overall test suite cost (as defined in Section 2.2.

A conducted case study reveals the effectiveness and the practicality of the proposed approach. In particular, it is capable of selecting test suites with higher pairwise coverage than randomly selected ones containing the same number of products. Additionally, it is able to generate test suites composed of less products and having a lower cost than randomly selected suites having the same pairwise coverage.

\section{A GENETIC ALGORITHM FOR MULTI- OBJECTIVE TEST GENERATION}

Exhaustive SPL testing is difficult to achieve in practice since it involves the test of thousands, even millions of products. As a result, it is necessary to select the products matching the testing objectives. Looking over the space of all the possible products for the optimal solution that satisfies the most these objectives is not feasible due to the large size of the space. Indeed, it is not possible to evaluate all the solutions. Genetic algorithms form a family of techniques that have been proven to be quite effective in finding solutions on large search spaces 11]. The approach introduced in this paper is a genetic algorithm that uses a SAT solver to only search the space of valid products, thus pruning the invalid ones.

\subsection{Genetic Algorithms}

Genetic algorithms form search-based heuristics mimicking the natural evolution process. They represent a smart way to randomly search for solutions to optimization problems. To apply such an approach, several parameters like the genes, the individuals and the objective function have to be defined. The individuals correspond to what composes a possible solution to the optimization problem. Each individual is composed of several units, called genes and the set of individuals that is handled by the algorithm is called the population. The objective function quantifies the individuals' ability to solve the optimization problem. Generally, these algorithms operate by repeatedly reproducing, adjusting and selecting the best individuals of the population. Based on the process presented in the following subsection, the population is gradually evolved by optimizing the solutions it encodes.

\subsubsection{The Process}

Genetic algorithms operate by evolving a population. The evolution is guided by an objective function. The initial population is usually produced at random and evolved based on a given set of operations on its individuals. Usually, three operations are used for the evolution of the population. These are the selection, crossover and mutation 11.

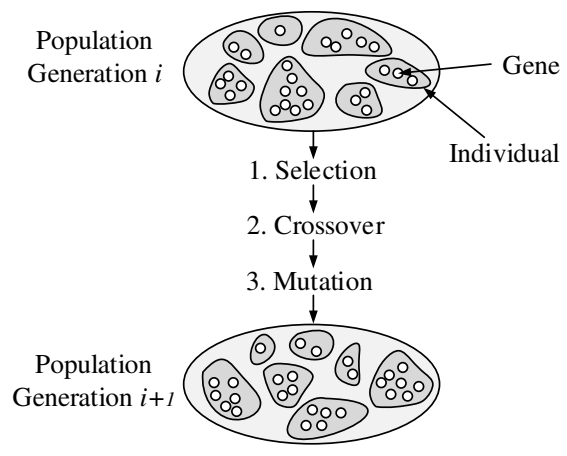

Figure 2: The process of evolving a population.

1. Selection chooses individuals for performing crossover and mutation. The selection is made by choosing the individuals with the best scores according to the objective function.

2. Crossover selects two individuals and switches some of their genes. This is usually performed by ordering the individuals' genes and switching all the genes after a randomly selected point. Crossover results into two new individuals called offsprings.

3. Mutation performs on an offspring by changing the values of one or more of its genes.

Performing the selection, crossover and mutation operations on a population results in one evolution cycle of the population. This cycle is called population generation. An overview of one generation is presented in Figure 2. The algorithm terminates after completing a predefined number of generations.

\subsubsection{Search for Multi-Objective Optimization}

Searching for optimizing more than one objective at the same time is usually referred to as multi-objective optimization. The aim of these approaches is to search for optimal (or nearly optimal) solutions requiring trade-offs between two or more conflicting objectives. In the present paper, our multi-objective optimization is defined as follows:

Let $X_{p}$ be the set of all the possible products of a SPL and let $x=\left\{P_{1}, \ldots, P_{m}\right\}$ be a set of $m$ products.

Given: a FM, a given amount of time or iterations, $t$, and the vector of $k$ objective functions $\mathbf{F}(\mathbf{x})=\left[F_{1}(x), \ldots, F_{k}(x)\right]^{T}$ where each objective function $F_{i}$ is a normalized function to minimize of the form $F_{i}(x): P_{1}, \ldots, P_{m} \rightarrow \mathbb{R}$.

Problem: finding $x \in X_{p}$ with respect to $t$ such as $\min _{\mathbf{x}} \mathbf{F}(\mathbf{x})$. The minimization of $\mathbf{F}$ is is the process of optimizing systematically and simultaneously the $k$ objective functions 20].

\subsection{Multi-Objective Test Generation for Soft- ware Product Lines}

The proposed approach is a multi-objective genetic algorithm. Like any genetic algorithm, it requires the definition of its ingredients (genes, individuals and population), its operations (selection, crossover and mutation) and the objective function that evaluates how each individual fits to the problem. 


\subsubsection{Modeling Individuals and Population}

A solution to our problem is a set of products that gives the maximum pairwise coverage with the minimum cost and number of products. To fit the problem with the genetic algorithm, it is needed to model the population, the individuals and the genes in terms of the actual problem. Therefore, since an individual represents a possible solution to the problem, it can be modeled as a set of products $x=\left\{P_{1}, \ldots, P_{m}\right\}$. Thus, each valid product represents a gene and the set of individuals handled by the genetic algorithm represents the population. This allows forming as a search space all the possible sets of valid products. This represents a huge space due to the intractable number of the possible products contained in a SPL.

However, enabling a search approach over this space cannot be performed directly. Recall that not all the products of a SPL form valid ones. Therefore, there is a need to efficiently deal with the invalid products. This is not an easy task due to the large number of invalid products, especially for large SPLs 25]. To overcome this difficulty, a SAT solver is used to provide random valid products. This is achieved by randomizing the solutions' enumeration order of the FM's formula [19]. To this end, random products, sets of random products and the initial population can be produced efficiently 12 . Thus, the search space is reduced to only include valid products. The importance of this step is that it prunes the invalid products from the search space.

\subsubsection{Modeling Genetic Algorithm Operations}

Crossover is an operation defined between two selected individuals, called the parents and it is performed as depicted by Figure 3 This operation is performed by selecting $l$ products from the smallest in size parent and swapping them with randomly selected ones from the other (bigger in size) parent. Our individuals form sets of products and thus the order of the genes does not matter. Hence, swapping randomly some products is equivalent to the usual crossover operation. Additionally, doing so ensures that the individuals are having the same sizes during the whole evolution process. Crossover operation results in two offsprings. These are then mutated according to the mutation operation as depicted by Figure 4. In mutation operation, a product is randomly selected from the individual and replaced by a randomly selected product (from the space of all the valid products).

Besides the above operations, the proposed approach incorporates two additional operations. These are the elitism and diversify operations. Elitism selects the best $e$ individuals of one population and includes them directly to the new one. Diversify operation adds one new individual, randomly produced directly into the new population. This ensures the

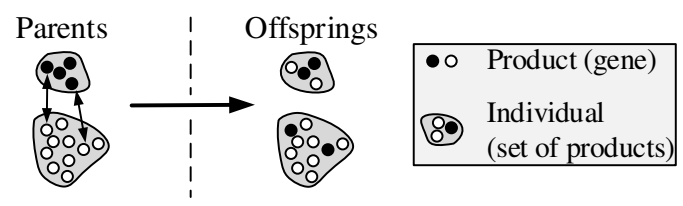

Figure 3: Crossover operation. A random number of $l$ products are selected in the smallest parent. Each of them is swapped with a random product selected from the other parent to produce the two offsprings.

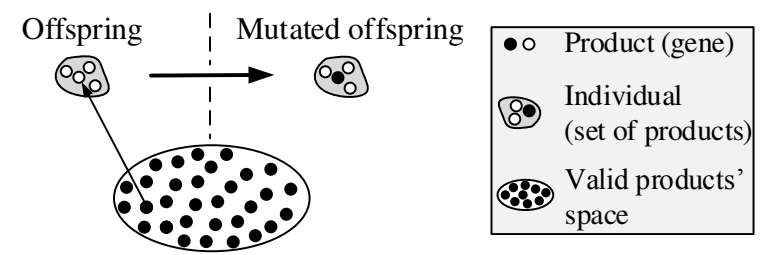

Figure 4: Mutation operation. A random product is selected from the offspring and replaced by a product randomly selected from the space of all the products valid towards the Feature Model.

diversity of the population individuals during the evolution process.

\subsubsection{The Objective Function}

The proposed approach is based on an objective function $\mathbf{F}(x)$, specially designed for the SPL testing context. As introduced in Section 3.1.2 $\mathbf{F}$ is a vector composed of the following $k=3$ objective functions $F_{1}, F_{2}$ and $F_{3}$.

1. Maximization of the pairwise coverage. This objective aims at ensuring that the selected products have the highest possible level of pairwise coverage:

$$
F_{1}(x)=\operatorname{cov}(x)
$$

where $c o v$ is a function that evaluates the number of pair of features covered by $x=\left\{P_{1}, \ldots, P_{m}\right\}$.

2. Minimization of the number of products. Here, the objective is to test the minimum number of products:

$$
F_{2}(x)=\operatorname{card}(x),
$$

where card is a function that returns the number of products $m$ of $x=\left\{P_{1}, \ldots, P_{m}\right\}$.

3. Minimization of the testing cost. This objective function aims at minimizing the cost of testing the products:

$$
F_{3}(x)=\operatorname{cost}(x),
$$

where cost is a function returning the cost of testing these $x=\left\{P_{1}, \ldots, P_{m}\right\}$ products.

In order to evaluate $\mathbf{F}$, each objective function is normalized so that they have the same magnitude using the following formula 20 :

$$
\frac{F_{i}(x)-F_{i}^{*}}{F_{i}^{\max }-F_{i}^{*}},
$$

where $F_{i}^{*}$ is the utopia point and $F_{i}^{\max }$ the maximum objective functions values. In addition, the objective $\max F_{1}$ is transformed into a minimization problem $\min \left(-F_{1}\right)$ in order to deal with minimization problems only. As a result, each objective function returns a value that holds between 0 and 1 , where 0 means that the objective is perfectly fulfilled. To evaluate $\mathbf{F}$, each function is assigned a weight $w_{j}$, where $\sum_{j=1}^{k} w j=1$. Thus, the fitness of each individual $I=\left\{P_{1}, \ldots, P_{m}\right\}$ is computed using a weighted sum as follows:

$$
\mathbf{F}(I)=\sum_{j=1}^{k} w_{j} F_{j}(I)
$$




\subsection{Algorithm}

The technique is formalized in Algorithm 1 . Informally, this approach starts by creating an initial random population (lines 3 to 14). The size of the population is specified by the user as long as the maximum size of an individual. Each individual is a set of $1, \ldots, m$ products randomly selected from the space of all the products that are valid towards the FM (lines 6 to 11). The objective function is then evaluated for each individual of the initial population (line 12).

The second step of the algorithm is the evolution of the population into a new one (lines 15 to 46). First, the elitism operation is performed (lines 17 to 19). Then, one random individual is created, evaluated and added to the new population to ensure having new products (lines 20 to 27). This is the diversity operation. The next steps ares crossover and mutation. To complete the new population until reaching its size $n$, individuals are created using crossover and mutation operators. The crossover (lines 29 and 30) aims at creating two offsprings from their selected parents. The two parents are selected using a fitness proportionate selection, also known as roulette wheel selection. The mutation occurs on the offsprings with a certain probability fixed by the user (lines 32 to 37 ). The fitness of these two offsprings is then evaluated and these two new individuals are added to the new population (lines 39 to 40 ).

Finally, the new population is reduced to the initial population size (lines 42 to 44 ) and the current population is replaced by the new one (line 45) and it continues to the next generation (line 46). When the algorithm terminates, the individual that has the best fitness, i.e. the one with the minimum $\mathbf{F}$ value is returned (line 47).

\section{CASE STUDY}

In this section, the proposed multi-objective test generation approach is assessed on a set of FMs. The objective of this case study is to answer the two following research questions:

- [RQ1] Is $\mathbf{F}$ capable of leading to a fulfilment of the three objectives? In other words, does the minimization of $\mathbf{F}$ results in a maximization of $F_{1}$ (or a mininimization of $\left(-F_{1}\right)$ ), a minimization of $F_{2}$ and a minimization of $F_{3}$ ?

- [RQ2] How does the multi-objective generation technique compares with a random one?

Answering the first question amounts to evaluate whether the objective function $\mathbf{F}$ is capable of improving the studied objectives. We expect to see a decreasing trend in all three objectives in relation to population generations. In practice, this means that a better trade-off can be achieved. This trade-off leads to a higher pairwise coverage, less products and a lower cost. Since no other approach takes into account these objectives at the same time, our second question aims at comparing the two of them when keeping the other one set, to enable the comparison with random test suite generation. Hence, we select random product sets of a) the same size and b) achieving the same pairwise coverage as our approach. If, for the same number of products, our approach achieves to provide a lower cost and higher pairwise coverage than the random set, we can consider it as being a better one. Similarly, it will be successful if it

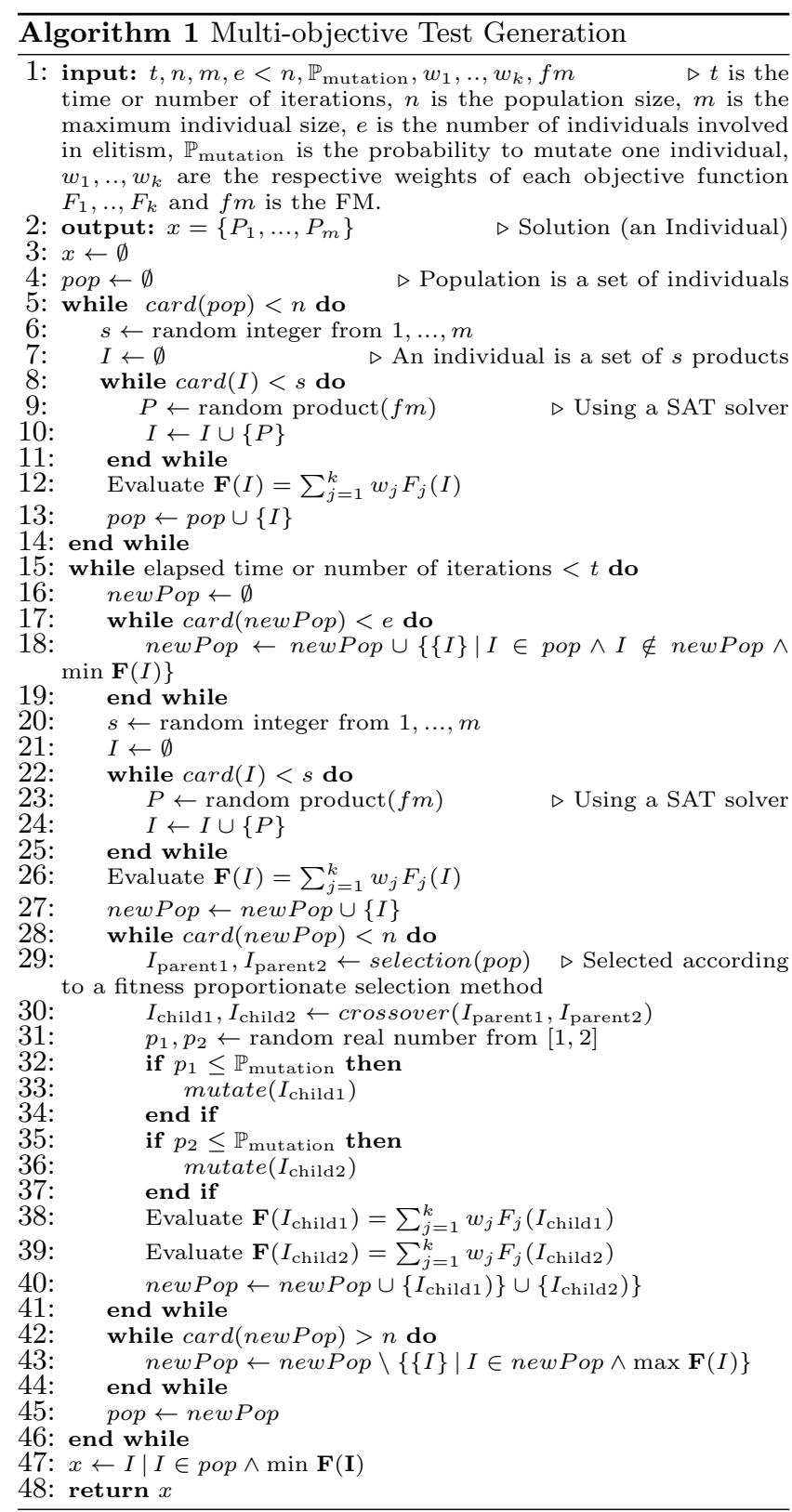

provides less products and a lower cost than random for a certain level of pairwise coverage.

To answer these questions, an experiment composed of 8 FMs of varying sizes was conducted. We applied our approach on these FMs to evaluate the population evolution and to compare it with a random approach. All the employed FMs were taken from the Software Product Line Online Tools (SPLOT) repository 22] and have been widely used in literature. The FMs details are recorded in Table 1 For each subject FM, the number of features, the number of products that can be configured and the number of valid pairs are presented. For each FM, we randomly assigned a value between 1 and 10 to all non-mandatory features to represent the cost value of the features, as presented in Section 2.2 Further details on the conducted experiment are given in the following subsections. 
Table 1: Feature Models used in the case study. It presents, for each of them, the number of features, the number of possible valid products that can be configured and the total number of valid pairs.

\begin{tabular}{|c|c|c|c|c|c|c|c|c|}
\hline & 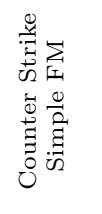 & 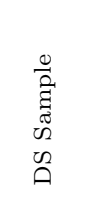 & 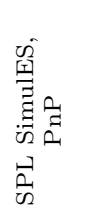 & 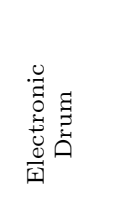 & 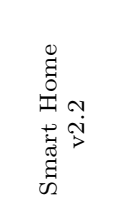 & 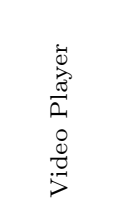 & 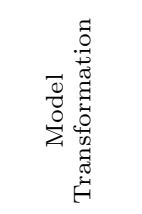 & 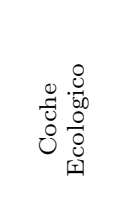 \\
\hline Number of features & 24 & 32 & 41 & 52 & 60 & 71 & 88 & 94 \\
\hline Number of products $(\approx)$ & 18,176 & 73,728 & 6,912 & 331,776 & $3.87 \times 10^{9}$ & $4.5 \times 10^{13}$ & $1.65 \times 10^{13}$ & $2.32 \times 10^{7}$ \\
\hline Number of pairs & 833 & 1,448 & 2,592 & 3,746 & 6,189 & 7,528 & 13,139 & 11,075 \\
\hline
\end{tabular}

\subsection{Algorithm Parameters}

Since objective $F_{1}$ results in selecting a higher number of products and $F_{2}, F_{3}$ results in selecting a lower number of products, we assigned the following weights: $w_{1}=0.5$ for $F_{1}$ and $w_{2}=w_{3}=0.25$ for $F_{2}$ and $F_{3}$. This assignment represents the balanced between the studied objectives as set for our experiment. It is noted that our approach is not limited to this balance. Thus, the tester may set a different balance according to his needs. The population size has been set to $n=100$ and the maximum size of an individual (a potential solution) has been set to $m=100$. The mutation probability $\mathbb{P}_{\text {mutation }}$ has been set up to 0.05 and the elitism value $e$ to 5 . Finally, the approach has been limited to run for $t=500$ generations.

\subsection{Evaluation of Objective Function F (RQ1)}

\subsubsection{Setup}

We performed the multi-objective test generation 30 times per FM using the above-mentioned parameters. For each of the 30 runs, we measured the initial values (at generation 1) and the final values (at generation 500) of both the 3 sub-objectives and the objective $\mathbf{F}$.

To evaluate whether these differences are statistically significant, we followed the guidelines suggested by Arcuri and Briand in [1] by performing a Mann-Whitney $U$ Test. It is a non-parametric statistical hypothesis test for assessing whether one of two samples of independent observations tends to have larger values than the other. We obtain from this test a probability called p-value which represents the probability that the two samples are equal. It is conventional in statistics to consider that the difference is not significant if the p-value is higher than the $5 \%$ level. The experiments involving this statistical test used two-tailed tests.

\subsubsection{Results}

Table 2 presents per FM the average values on the 30 runs for each of the objective and for $\mathbf{F}$. $F_{1}$ is the number of pairs covered by the generated products (to maximize), $F_{2}$ is the number of products (to minimize) and $F_{3}$ is the cost of testing the generated products (to minimize). $\mathbf{F}$ is the compromised between the 3 objectives. From this table, one may observe that final values of both the 3 objectives and the objective function are better than initial one, i.e. decreasing for $F_{2}, F_{3}$ and $\mathbf{F}$ and increasing for $F_{1}$ since it's a maximization. This underlines that a decreasing in $\mathbf{F}$ leads to a better fulfillment of each objective. These difference are most of the time statistically significant with p-values lower than 0.05 or highly significant with p-values lower than 0.001 , fact which demonstrates the appropriateness of the objective function with only 500 generations of the algorithm.

Besides, Figure 5 depicts the evolution of the objective function $\mathbf{F}$ and the normalized objective function over the generations of the algorithm. Since all the three objectives are transformed into minimization problems, i.e. lower values of the objective functions represent better solutions to the problem, this figure clearly shows the decreasing trend of each objective function. It therefore demonstrates that $\mathbf{F}$ leads to a better solution regarding all the examined objectives.

\subsubsection{Answering RQ1}

The results presented in the previous section clearly show the ability of the objective function to fulfill the three studied objectives. In particular, $\mathbf{F}$ is capable of finding better solutions for all the objectives under investigation. While some differences may not be statistically significant, recall that the approach is a compromise between the conflicting objectives. It therefore tends to compromise the 3 objectives according the $w_{1}, w_{2}$ and $w_{3}$ parameters. The overall

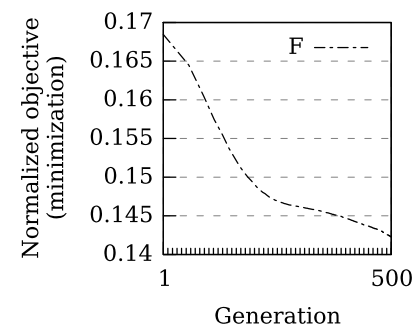

(a) $\mathbf{F}$

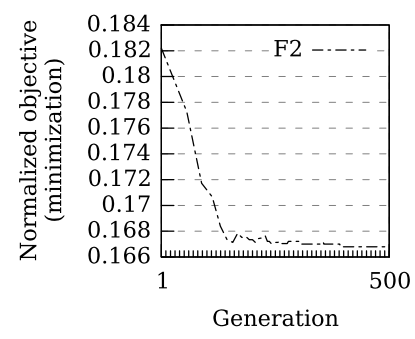

(c) $F_{2}$

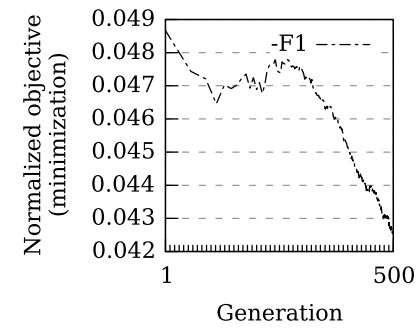

(b) $-F_{1}$

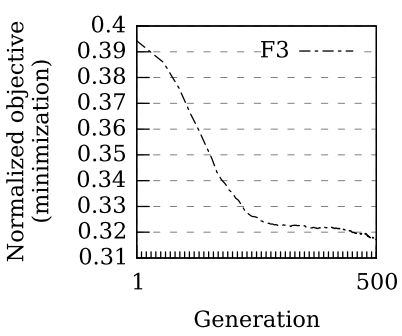

(d) $F_{3}$
Figure 5: Evolution of the objective function $F$ and each normalized sub-objectives (to be minimized) during the 500 generations of the multi-objective test generation approach. 
Table 2: Evolution of the objective function $F$ and the sub-objectives from the initial generation of the multiobjective approach to the final one (500 generations). The final and initial values are the average between the 30 runs. The p-value is the results of the Mann-Whitney $U$ Test between the 30 first initial values and the 30 final ones.

\begin{tabular}{|c|c|c|c|c|c|c|c|c|c|c|c|c|}
\hline & \multicolumn{3}{|c|}{$\mathbf{F}$} & \multicolumn{3}{|c|}{$F_{1}$ : pairwise coverage (to maximize) } & \multicolumn{3}{|c|}{$F_{2}: \#$ products (to minimize) } & \multicolumn{3}{|c|}{$F_{3}:$ cost (to minimize) } \\
\hline & Initial & Final & p-value & Initial & Final & p-value & Initial & Final & p-value & Initial & Final & p-value \\
\hline Counter Strike Simple FM & 0.163 & 0.115 & $<0.001$ & 819.7 & 819.63 & 0.79 & 15.46 & 14.466 & 0.176 & 658.13 & 369.90 & $<0.001$ \\
\hline SPL SimulES, PnP & 0.163 & 0.136 & $<0.001$ & 1431.4 & 1439.03 & 0.003 & 14.33 & 12.8 & $<0.001$ & 906.73 & 680.66 & $<0.001$ \\
\hline DS Sample & 0.189 & 0.172 & $<0.001$ & 2364.2 & 2382.9 & 0.07 & 31.866 & 27.7 & $<0.001$ & 1040.2 & 887.96 & $<0.001$ \\
\hline Electronic Drum & 0.146 & 0.132 & $<0.001$ & 3633.6 & 3665.06 & $<0.001$ & 18.7 & 17.4 & 0.04 & 1221.96 & 1079.6 & 0.001 \\
\hline Smart Home v2.2 & 0.177 & 0.138 & $<0.001$ & 6041.46 & 6056.66 & 0.60 & 17.7 & 17.03 & 0.33 & 2282.86 & 1537.46 & $<0.001$ \\
\hline Video Player & 0.162 & 0.135 & $<0.001$ & 7430.66 & 7428.76 & 0.20 & 15.13 & 13.86 & 0.011 & 2000.63 & 1443.86 & $<0.001$ \\
\hline Model Transformation & 0.175 & 0.153 & $<0.001$ & 12733.73 & 12788.1 & 0.387 & 17.96 & 17.16 & 0.48 & 3522.5 & 2829.36 & $<0.001$ \\
\hline Coche Ecologic & 0.169 & 0.154 & $<0.001$ & 10560.26 & 10618.06 & 0.039 & 21.13 & 19.66 & 0.19 & 2083.1 & 1761.63 & $<0.001$ \\
\hline
\end{tabular}

objective $\mathbf{F}$ has always highly statistically significance difference, showing that $\mathbf{F}$ clearly guides the population generation. Finally, it must be mentioned that the proposed approach achieves the above results using only a small number of generations (500 generations). This can be viewed as an achievement of the approach since search-based approaches do require thousands of executions in order to be effective 11.

\subsection{Comparison with Random (RQ2)}

\subsubsection{Setup}

To assess our approach, we compared it with a baseline. To do so, we used two baseline comparison basis. In the first one, we selected random sets of products having the same $F_{1}$ value as our approach. In the second one we selected random product sets having the same $F_{2}$ value as our approach. The $F_{1}$ comparison basis aims at evaluating how many products for which cost are provided by the examined approaches (baseline and proposed) to achieve the same level of pairwise coverage. The $F_{2}$ comparison basis evaluates the pairwise coverage and the cost induced by the generated products for the same number of products. In the end, for each run of our approach, two random runs have been performed: the first one by setting $F_{1}$ as the comparison basis and the second one using $F_{2}$. The conducted experiment (including both the baseline and the proposed approach) was independently repeated 30 times.

To evaluate whether the differences are significant, we performed a Mann-Whitney U Test, as presented in Section 4.2.1 For each comparison $\left(\mathbf{F}, F_{2}\right.$ and $F_{3}$ on $F_{1}$ comparison basis and $\mathbf{F}, F_{1}$ and $F_{3}$ on $F_{2}$ comparison basis), we got one p-value per FM, i.e. 8 in the total. Each p-value results from the comparison between the 30 values obtained on the 30 runs by the proposed approach with those obtained at random.

\subsubsection{Results}

Table 3 records the comparison between our test generation approach and the baseline one based on the $F_{1}$ and the $F_{2}$ comparison basis. For each FM and each comparison basis, the average, minimum and maximum values of $\mathbf{F}$ and the three objectives $F_{1}, F_{2}$ and $F_{3}$ one the 30 runs are presented. $F_{1}$ represents the number of pairs covered by the generated products (to be maximized), $F_{2}$ represents the number of products (to be minimized) and $F_{3}$ represents the cost of testing the generated products (to be minimized). From this table, it is clear that the proposed approach performs better than a random one. For instance, for the Smart Home v2.2 FM on the $F_{1}$ comparison basis (i.e. for achieving the same pairwise coverage), the proposed approach proposes on average around 17 products with a cost of $\approx 1,537$ where a random technique requires around 22 products with a cost of $\approx 3,016$.

Figure 6 depicts the achieved values for each objective for both basis of comparison. The values are the average on all the FMs for the 30 runs. Here, the smallest triangle signifies a better solution since each normalized objective is a function to be minimized. An objective value equals to 0 means that this objective is perfectly fulfilled. The length of the axis of each objective is 1 . This figure shows that a) for the same pairwise coverage, the proposed approach requires less products with a lower cost and b) for the same number of products, our approach provides a higher pairwise coverage and a lower cost compared to random products.

Finally, the results of the statistical test are depicted by Figure 7 It presents, for each comparison, the distribution of the 8 p-values (one per FM). Each p-value is the result of the comparison between the 30 values obtained for each objective during each run of the proposed and random approaches. From this figure, one may observe that all the p-values are lower to 0.001 , fact which denotes the a high statistical difference between the results achieved by our approach compared to the results of the random one.

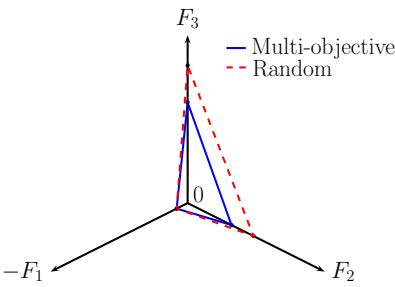

(a) Same pairwise coverage $\left(F_{1}\right.$ basis $)$

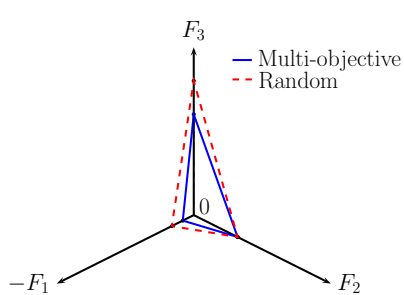

(b) Same number of products $\left(F_{2}\right.$ basis $)$
Figure 6: Normalized sub-objectives (to be minimized) according to $F_{1}$ and $F_{2}$ comparison basis. Values closer to 0 represent better solutions. These values are the average on all the FMs for all the 30 runs of each approach. The length of each axis is 1 . 
Table 3: Comparison between the multi-objective test generation approach and the random one. For each Feature Model, the values of the objective functions studied and $\mathbf{F}$ are represented. $F_{1}$ is the number of pairs (to be maximized), $F_{2}$ is the number of products (to be minimized) and $F_{3}$ is the cost (to be minimized). The comparison with random has been made by fixing either $F_{1}$ or $F_{2}$ on 30 runs per approach.

\begin{tabular}{|c|c|c|c|c|c|c|c|c|}
\hline & & & \multirow{2}{*}{\multicolumn{3}{|c|}{ Multi-objective test generation }} & \multirow{2}{*}{\multicolumn{3}{|c|}{ Random test generation }} \\
\hline & & & & & & & & \\
\hline & & & avg & $\min$ & $\max$ & avg & $\min$ & $\max$ \\
\hline \multirow{6}{*}{ Counter Strike Simple FM } & \multirow{3}{*}{ Same pairwise coverage ( $F_{1}$ basis) } & $\mathbf{F}$ (to $\min )$. & 0.115 & 0.107 & 0.122 & 0.173 & 0.154 & 0.2 \\
\hline & & $F_{2}$ (to $\left.\min .\right)$ & 14.466 & 11 & 17 & 18.833 & 12 & 28 \\
\hline & & $F_{3}$ (to min.) & 369.9 & 276 & 440 & 832.433 & 512 & 1,218 \\
\hline & \multirow{3}{*}{ Same number of products ( $F_{2}$ basis) } & $\mathbf{F}$ (to $\min )$. & 0.115 & 0.107 & 0.122 & 0.181 & 0.155 & 0.238 \\
\hline & & $F_{1}$ (to max.) & 819.633 & 803 & 828 & 806.833 & 763 & 828 \\
\hline & & $F_{3}$ (to min.) & 369.9 & 276 & 440 & 665.13 & 510 & 889 \\
\hline \multirow{6}{*}{ SPL SimuelES, PnP } & \multirow{3}{*}{ Same pairwise coverage ( $F_{1}$ basis) } & $\mathbf{F}$ (to $\min )$. & 0.136 & 0.13 & 0.14 & 0.173 & 0.159 & 0.187 \\
\hline & & $F_{2}$ (to $\left.\min .\right)$ & 12.8 & 10 & 15 & 18.966 & 14 & 26 \\
\hline & & $F_{3}$ (to $\left.\min .\right)$ & 680.666 & 567 & 813 & $1,234.8$ & 940 & 1,656 \\
\hline & \multirow{3}{*}{ Same number of products ( $F_{2}$ basis) } & $\mathbf{F}$ (to $\min )$. & 0.136 & 0.13 & 0.14 & 0.177 & 0.16 & 0.20 \\
\hline & & $F_{1}$ (to $\left.\max .\right)$ & $1,439.033$ & 1,429 & 1,446 & $1,411.066$ & 1,367 & 1,445 \\
\hline & & $F_{3}$ (to $\left.\min .\right)$ & 680.666 & 567 & 813 & 859.333 & 680 & 1,039 \\
\hline \multirow{6}{*}{ DS Sample } & \multirow{3}{*}{ Same pairwise coverage ( $F_{1}$ basis) } & $\mathbf{F}$ (to $\min )$. & 0.172 & 0.169 & 0.177 & 0.214 & 0.182 & 0.302 \\
\hline & & $F_{2}$ (to $\left.\min .\right)$ & 27.7 & 22 & 34 & 44.966 & 32 & 83 \\
\hline & & $F_{3}$ (to $\min$.) & 887.966 & 700 & 1,106 & $1,469.8$ & 1,024 & 2,716 \\
\hline & \multirow{3}{*}{ Same number of products ( $F_{2}$ basis $)$} & $\mathbf{F}$ (to min.) & 0.172 & 0.169 & 0.177 & 0.214 & 0.195 & 0.246 \\
\hline & & $F_{1}($ to $\max )$. & $2,382.9$ & 2,328 & 2,428 & $2,236.366$ & 2,093 & 2,362 \\
\hline & & $F_{3}$ (to min.) & 887.966 & 700 & 1,106 & 902.9 & 725 & 1,121 \\
\hline \multirow{6}{*}{ Electronic Drum } & \multirow{3}{*}{ Same pairwise coverage ( $F_{1}$ basis) } & $\mathbf{F}$ (to $\min )$. & 0.132 & 0.130 & 0.133 & 0.155 & 0.142 & 0.173 \\
\hline & & $F_{2}$ (to $\left.\min .\right)$ & 17,4 & 14 & 20 & 24.5 & 17 & 32 \\
\hline & & $F_{3}$ (to $\left.\min .\right)$ & $1,079.6$ & 872 & 1,255 & $1,645.533$ & 1.165 & 2,210 \\
\hline & \multirow{3}{*}{ Same number of products ( $F_{2}$ basis $)$} & $\mathbf{F}$ (to $\min )$. & 0.132 & 0.130 & 0.133 & 0.155 & 0.144 & 0.180 \\
\hline & & $F_{1}$ (to $\left.\max .\right)$ & 3,665 & 3,628 & 3,693 & $3,585.133$ & 3,458 & 3,661 \\
\hline & & $F_{3}$ (to $\left.\min .\right)$ & $1,079.6$ & 872 & 1,255 & $1,174.7$ & 926 & 1,367 \\
\hline \multirow{6}{*}{ Smart Home v2.2 } & \multirow{3}{*}{ Same pairwise coverage ( $F_{1}$ basis) } & $\mathbf{F}$ (to $\min )$. & 0.138 & 0.133 & 0.144 & 0.191 & 0.166 & 0.234 \\
\hline & & $F_{2}$ (to $\left.\min .\right)$ & 17.033 & 12 & 20 & 21.966 & 15 & 36 \\
\hline & & $F_{3}$ (to $\left.\min .\right)$ & $1,537.466$ & 1,195 & 1,836 & $3,016.533$ & 1,974 & 5,184 \\
\hline & & $\mathbf{F}$ (to $\min )$. & 0.138 & 0.133 & 0.144 & 0.19 & 0.166 & 0.223 \\
\hline & Same number of products ( $F_{2}$ basis $)$ & $F_{1}$ (to max.) & $6,056.666$ & 5,973 & 6,107 & 5,976 & 5,756 & 6,087 \\
\hline & & $F_{3}$ (to min.) & $1,537.466$ & 1,195 & 1,836 & $2,330.4$ & 1,532 & 2,872 \\
\hline & & $\mathbf{F}$ (to $\min )$. & 0.135 & 0.128 & 0.138 & 0.167 & 0.152 & 0.188 \\
\hline & Same pairwise coverage ( $F_{1}$ basis) & $F_{2}$ (to $\left.\min .\right)$ & 13.866 & 11 & 16 & 16.5 & 14 & 24 \\
\hline Video Player & & $F_{3}$ (to min.) & $1,443.866$ & 1,230 & 1,687 & $2,2236.5$ & 1,858 & 3,339 \\
\hline & & $\mathbf{F}$ (to min.) & 0.135 & 0.128 & 0.138 & 0.173 & 0.159 & 0.208 \\
\hline & Same number of products ( $F_{2}$ basis) & $F_{1}$ (to $\left.\max .\right)$ & $7,428.766$ & 7,739 & 7,468 & $7,341.233$ & 6,925 & 7.471 \\
\hline & & $F_{3}$ (to min.) & $1,443.866$ & 1,230 & 1,687 & 1907.433 & 1,467 & 2,444 \\
\hline & & $\mathbf{F}$ (to $\min )$. & 0.153 & 0.149 & 0.158 & 0.185 & 0.176 & 0.199 \\
\hline & Same pairwise coverage ( $F_{1}$ basis) & $F_{2}$ (to $\left.\min .\right)$ & 17.166 & 14 & 21 & 20,733 & 18 & 25 \\
\hline Model Transformation & & $F_{3}$ (to $\left.\min .\right)$ & $2,829.366$ & 2,353 & 3,319 & $4,325.166$ & 3,588 & 5,304 \\
\hline Model Transformation & & $\mathbf{F}$ (to $\min )$. & 0.153 & 0.149 & 0.158 & 0.187 & 0.174 & 0.199 \\
\hline & Same number of products ( $F_{2}$ basis) & $F_{1}$ (to $\left.\max .\right)$ & $12,788.1$ & 12,657 & 12,902 & $12,595.3$ & 12,262 & 12,815 \\
\hline & & $F_{3}$ (to $\left.\min .\right)$ & $2,829.366$ & 2,353 & 3,319 & $3,556.166$ & 2,742 & 4,509 \\
\hline & & $\mathbf{F}$ (to $\min )$. & 0.154 & 0.151 & 1.158 & 0.186 & 0.172 & 0.217 \\
\hline & Same pairwise coverage ( $F_{1}$ basis $)$ & $F_{2}$ (to $\left.\min .\right)$ & 19.666 & 16 & 24 & 29.3 & 20 & 41 \\
\hline Coche Frolooico & & $F_{3}$ (to min.) & $1,761.633$ & 1383 & 2153 & $2,984,3$ & 2,051 & 4,384 \\
\hline Coche Ecologico & & $\mathbf{F}$ (to min.) & 0.154 & 0.151 & 1.158 & 0.188 & 0.167 & 0.207 \\
\hline & Same number of products ( $F_{2}$ basis) & $F_{1}$ (to $\left.\max .\right)$ & 10,618 & 10,492 & 10,726 & 10,302 & 10,040 & 10,553 \\
\hline & & $F_{3}$ (to $\left.\min .\right)$ & $1,761.633$ & 1383 & 2153 & $2,016.333$ & 1,631 & 2,404 \\
\hline
\end{tabular}

\subsubsection{Answering $R Q 2$}

We compared the multi-objective test generation approach with a baseline technique using $F_{1}$ and $F_{2}$ as a basis comparison. In all the cases, the objectives are better fulfilled by our approach, as demonstrated by the results of Section 4.3 .2 Overall, for the same pairwise coverage, the approach selects less products with a lower cost. For the same number of products, the approach provides a higher pairwise coverage at a lower cost. In addition, the differences between the objectives values reach by our technique and the values reached by the baseline are statistically highly significant, fact which demonstrates the effectiveness of the approach. 


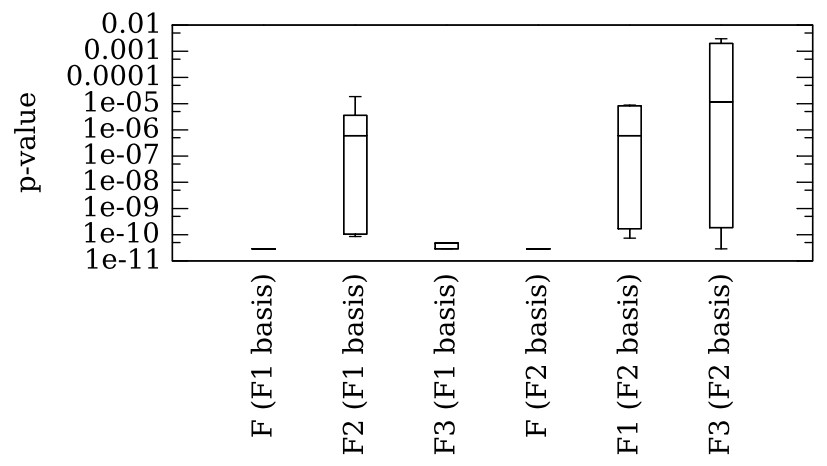

Figure 7: Distribution of the p-values for the comparison with random. For each comparison $\left(\mathbf{F}, F_{2}\right.$ and $F_{3}$ on $F_{1}$ comparison basis and $\mathbf{F}, F_{1}$ and $F_{3}$ on $F_{2}$ comparison basis), the 8 p-values (one per Feature Model) are represented with a boxplot. Each p-value has been obtained by comparing the 30 values obtained on the 30 runs for each approach.

\subsubsection{Threats to Validity}

The conducted experiments involve potential threats to validity. First, there is a threat regarding the generalization of the results reported in this study. Indeed, a different set of FMs might output different results. We used a set of 8 FMs widely used in literature with different size and level of complexity to reduce this threat and to ensure that the FMs used form a good sample.

Additional threats can be identified due to a) to our implementation, which might contains errors that can affect the presented results and $b$ ) the performed experiments. To overcome this issue, we divided our implementation into subroutines to minimize the potential errors and we make it publicly available. We also repeated the conducted experiments independently for 30 times to avoid any risk due to random effects, like the fortunate selection of the (nearest) optimal solution.

\section{RELATED WORK}

Test generation is an issue than has been investigated by the research community since the last decades 8.

With respect to constraint solving, Johansen et al. 15 proposed a covering array technique to generate the products covering all the $t$-wise interactions between features. In the same lines, a search-based approach that achieves scalable but partial $t$-wise coverage has been introduced in 12 . Perrouin et al. 26] proposed a method based on the Alloy SAT solver to generate $t$-wise test suites. Modeling the importance of $t$-wise interactions have been proposed in 16 by putting weights on interactions and measuring weight coverage. Like the approach presented in this paper, these methods have been applied in conjunction with a SAT solver but aim at fulfilling only one objective at a time, i.e. either the $t$-wise coverage or the weight coverage. Other work, e.g. 13 , 14 used a SAT solver to only generate valid products. Here, maximizing the 2 -wise coverage of the generated products is one of the 3 objectives to fulfill simultaneously. Moreover, we combine constraint solving with search-based techniques.

Regarding evolutionary algorithms, Konak et al. 18, proposed a tutorial on the use of these kind of methods for multi-objective optimization purposes. Ensan et al. 9 proposed a genetic algorithm approach where each gene is a feature. The crossover can thus produce invalid products. Furthermore, the explored space may contain invalid products. Their fitness function measures coverage by evaluating the variability points to be bound and the constraints concerned by the features of a product. In our approach, we use a SAT solver to only explore the space containing products valid towards the FM. The modeling of genes is performed at the product level and the crossover and mutation operators introduced avoid the introduction of invalid products.

Finally, in the context of multi-objective optimization, Olaechea et al. 24. introduced a tool that supports multiobjective goals in the configuration of features. The approach works on attributed feature models which provide quality attributes to features. Their technique use an exact solving of the multi-optimization problem and consider FMs with one to 3 objectives, where the one with 3 objectives contains 12 features. In our approach, we use an heuristic solving with 3 objectives to fulfill for all the FMs. The advantage is that it allows to scale to large FMs.

\section{CONCLUSIONS AND FUTURE WORK}

Optimizing different objectives is a hard problem due to the presence of conflicts between them. For example, minimizing the number of tests is in conflict with the maximization of their pairwise coverage since generally more tests lead to a higher coverage. To tackle this problem, the present paper introduces a multi-objective genetic algorithm specially adapted for SPLs. Our approach combines genetic algorithms and constraint solving techniques in a complementary way. Thus, it provides sets of products to test that simultaneously optimize pairwise coverage and testing costs. The work presented in this paper deals with the following issues:

- Model the test generation problem for SPLs as a search problem. The proposed approach models products as genes and sets of products as individuals. It also suggests some possible operations on the individuals and an objective function. Therefore, it enables search-based approaches to solve the test generation problem.

- Use constraint solving technique to prune the invalid products from the search space. This is a crucial step towards enabling an efficient search process. Our initial experiments show that it is almost impossible to construct sets of valid products without using a constraint solver. Even if this is possible, since invalid products must be removed from the final product sets, they only add barriers to the search process. Thus, it is clear that removing the invalid product greatly reduces the search space and busts the effectiveness of the approach.

- Propose a genetic algorithm to solve the multiobjective optimization problem. We propose a generic algorithm for handling the test generation problem according multiple objectives for the context of SPLs. The conducted study show that the approach is practically effective and feasible.

Reproducible tests has been identified as a central tenet of testing [10]. Therefore, to enable the reproducibility of our results, we make the source code of our approach and the data used for the experiments publicly available at: 
http://research.henard.net/SPL/SPLC_2013/

Finally, future work includes the following points:

- Conducting additional experiments to further validate the finding of the present paper.

- Investigating the effects of the parameters on the effectiveness and efficiency of the proposed approach.

- Investigating criteria for deciding when to stop the evolution process.

- Applying our approach on real and large scale subjects in order to better quantify the benefits of the approach.

\section{Acknowledgment}

The present work is supported by the Fonds National de la Recherche (FNR), Luxembourg, under the projects MITER C10/IS/783852 and MODEL C12/IS/3977071.

\section{REFERENCES}

[1] A. Arcuri and L. Briand. A practical guide for using statistical tests to assess randomized algorithms in software engineering. In ICSE, pages 1-10, 2011.

[2] D. Benavides, S. Segura, and A. Ruiz-Cortés. Automated analysis of feature models 20 years later: A literature review. Inf. Syst., 35(6):615-636, Sept. 2010.

[3] P. Clements and L. Northrop. Software Product Lines: Practices and Patterns. 2001.

[4] D. M. Cohen, S. R. Dalal, M. L. Fredman, and G. C. Patton. The AETG System: an approach to testing based on combinatorial design. IEEE Transactions on Software Engineering, 23(7):437-444, 1997.

[5] J. Coplien, D. Hoffman, and D. Weiss. Commonality and variability in software engineering. IEEE Softw., 15(6):37-45, Nov. 1998.

[6] K. Czarnecki and U. W. Eisenecker. Generative programming: methods, tools, and applications. 2000.

[7] H. Do, S. Mirarab, L. Tahvildari, and G. Rothermel. An empirical study of the effect of time constraints on the cost-benefits of regression testing. In FSE, pages 71-82, 2008.

[8] I. do Carmo Machado, J. D. McGregor, and E. Santana de Almeida. Strategies for testing products in software product lines. SIGSOFT Softw. Eng. Notes, 37(6):1-8, Nov. 2012.

[9] F. Ensan, E. Bagheri, and D. Gašević. Evolutionary search-based test generation for software product line feature models. In CAiSE, pages 613-628, 2012.

[10] M. D. Ernst. Reproducible tests? non-duplicable results in testing and verification. In ICST, April 18-20, 2012.

[11] M. Harman and P. McMinn. A theoretical and empirical study of search-based testing: Local, global, and hybrid search. IEEE Trans. Softw. Eng., 36(2):226-247, Mar. 2010.

[12] C. Henard, M. Papadakis, G. Perrouin, J. Klein, P. Heymans, and Y. L. Traon. Bypassing the combinatorial explosion: Using similarity to generate and prioritize t-wise test suites for large software product lines. CoRR, abs/1211.5451, 2012.
[13] C. Henard, M. Papadakis, G. Perrouin, J. Klein, and Y. Le Traon. Assessing software product line testing via model-based mutation: An application to similarity testing. In ICSTW, A-MOST, 2013.

[14] C. Henard, M. Papadakis, G. Perrouin, J. Klein, and Y. Le Traon. Towards automated testing and fixing of re-engineered feature models. In ICSE, pages 1245-1248, 2013.

[15] M. F. Johansen, O. Haugen, and F. Fleurey. An algorithm for generating t-wise covering arrays from large feature models. In $S P L C$, pages 46-55, 2012.

[16] M. F. Johansen, Ø. Haugen, F. Fleurey, A. G. Eldegard, and T. Syversen. Generating better partial covering arrays by modeling weights on sub-product lines. In MoDELS, pages 269-284, 2012.

[17] K. Kang, J. Lee, and P. Donohoe. Feature-oriented product line engineering. Software, IEEE, 19(4):58 65, jul/aug 2002.

[18] A. Konak, D. W. Coit, and A. E. Smith. Multi-objective optimization using genetic algorithms: A tutorial. Reliability Engineering \& System Safety, 91(9):992-1007, Sept. 2006.

[19] D. Le Berre and A. Parrain. The sat4j library, release 2.2, system description. Journal on Satisfiability, Boolean Modeling and Computation(JSAT), 7:59-64, 2010.

[20] R. T. Marler and J. S. Arora. Survey of multi-objective optimization methods for engineering. Structural and Multidisciplinary Optimization, 26(6):369-395, Apr. 2004.

[21] J. McGregor. Testing a software product line. In Testing Techniques in Software Engineering, volume 6153, pages 104-140. 2010.

[22] M. Mendonca, M. Branco, and D. Cowan. S.p.l.o.t.: software product lines online tools, 2009.

[23] M. Mendonca, A. Wasowski, and K. Czarnecki. Sat-based analysis of feature models is easy. In $S P L C$, pages 231-240, 2009.

[24] R. Olaechea, S. Stewart, K. Czarnecki, and D. Rayside. Modelling and multi-objective optimization of quality attributes in variability-rich software. In NFPinDSML, pages 2:1-2:6, 2012.

[25] G. Perrouin, S. Oster, S. Sen, J. Klein, B. Baudry, and Y. L. Traon. Pairwise testing for software product lines: comparison of two approaches. Software Quality Journal, 20(3-4):605-643, 2012.

[26] G. Perrouin, S. Sen, J. Klein, B. Baudry, and Y. Le Traon. Automated and scalable t-wise test case generation strategies for software product lines. In ICST, pages 459-468, 2010.

[27] K. Pohl, G. Böckle, and F. J. v. d. Linden. Software Product Line Engineering: Foundations, Principles and Techniques. 2005.

[28] R. Rabiser, P. Grunbacher, and D. Dhungana. Supporting product derivation by adapting and augmenting variability models. In $S P L C$, pages 141-150, 2007.

[29] S. Yoo and M. Harman. Regression testing minimization, selection and prioritization: a survey. Software Testing, Verification and Reliability, 22(2):67-120, 2012 